Originalien

Unfallchirurg 2021 124:311-318

https://doi.org/10.1007/s00113-020-00903-6

Angenommen: 16 . September 2020

Online publiziert: 27 . Oktober 2020

(c) Der/die Autor(en) 2020

\section{Redaktion}

W. Mutschler, München

H. Polzer, München

B. Ockert, München

N. Moellhoff' $\cdot$ H. Polzer ${ }^{2}$ S. F. Baumbach ${ }^{2} \cdot$ K. G. Kanz ${ }^{3} \cdot$ W. Böcker ${ }^{2} \cdot$ V. Bogner-

\title{
Flatz $^{2}$
}

${ }^{1}$ Abteilung für Hand-, Plastische und Ästhetische Chirurgie, Klinikum der Universität München, LMU

München, München, Deutschland

${ }^{2}$ Klinik für Allgemeine, Unfall- und Wiederherstellungschirurgie, Klinikum der Universität München, LMU München, München, Deutschland

${ }^{3}$ Klinik und Poliklinik für Unfallchirurgie, Klinikum rechts der Isar, Technische Universität München, München, Deutschland

\section{Unguis incarnatus - konservative oder operative Therapie? Ein praktischer Behandlungsalgorithmus}

schließen den Nagel seitlich ein. Das Nagelbett dient als Gleit- und Haftlager für den Nagel. Es wird distal vom Nagelisthmus begrenzt, der in das Hyponychium unter dem freien Nagelrand übergeht [3].

Der Unguis incarnatus ist eine der häufigsten Erkrankung des Nagelorgans [4, 5]. Die Prävalenz beträgt zwischen 2,5 und $5 \%[6,7]$, mit einer Häufung im Jugend- und im jungen Erwachsenenalter. Männer sind dabei etwa doppelt so häufig wie Frauen betroffen.

Ein eingewachsener Zehennagel entsteht, wenn die Nagelplatte die periunguale Haut irritiert. Dies führt im Sinne einer Fremdkörperreaktion zu Inflammation und Hypergranulation mit sekundärer Infektion des betroffenen $\mathrm{Na}$ gelwalls. Klinisch präsentiert sich der Patient mit Schmerzen, Rötung und Schwellung des Nagelwalls oder des gesamten Zehs. Eine Abszedierung kann vorliegen. Meist ist die lateralseitige Großzehe betroffen [7-9].

Die häufigsten Ursachen für den Unguis incarnatus, v. a. bei jungen Patienten, sind das falsche Schneiden der Nägel und das Tragen von zu engem Schuhwerk. Durch falsches rundes Zurückschneiden des Nagels kann im Bereich des seitlichen Nagelwalls eine spitze Nagelecke - auch Spikulum genannt - entstehen, die folglich in den Nagelwall einwächst. Enges Schuhwerk führt zu einem ver- mehrten interdigitalen Druck zwischen Hallux und zweiter Zehe. Dadurch wird bei der Großzehe die laterale Nagelplatte gegen den lateralen Nagelwall gedrückt, was wiederrum $\mathrm{zu}$ einer lokalen Irritation führen kann. Weitere Ursachen sind mangelnde Nagelpflege/Hygiene, wiederholte Traumata am Nagelorgan, Hyperhidrose, Nagelpilzbefall, Diabetes mellitus, Immunsuppression, Schwangerschaft, die Einnahme von EGF-Rezeptor-Inhibitoren oder mechanische Ursachen durch Fußfehlstellungen [7, 10-13]. Auch Hyperostosen bzw. Osteophyten können durch Druck auf die Nagelmatrix oder das Nagelbett zu Nagelfehlbildungen („pincer nail“) und sekundären Irritationen führen [14]. Des Weiteren ist eine Reihe endogener Ursachen beschrieben: Nageldeformitäten wie die Onychogrypose [15] und anlagebedingte anatomische Varianten können einen Unguis incarnatus begünstigen. Eine positive Familienanamnese ist dann in den meisten Fällen zu beobachten [5, 16].

Der Unguis incarnatus ist ein häufiges Krankheitsbild, mit welchem verschiedenste medizinische Fachrichtungen konfrontiert sind. Trotz der erstaunlichen Menge an Literatur fehlt ein differenzierter Diagnose- und Behandlungsalgorithmus [17, 18]. Im Rahmen dieser Arbeit soll die vorhandene Evidenz liegenden Nagel. Die lateralen Nagelwälle 


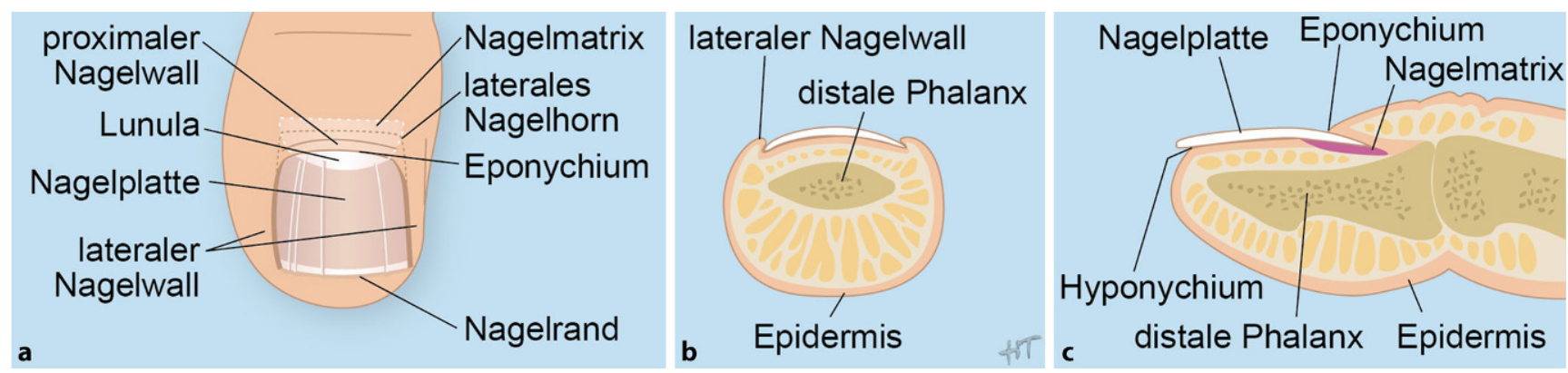

Abb. 1 A Anatomie der Großzehe. a Von oben, b im Querschnitt, c im Längsschnitt. (Mit freundl. Genehmigung der Autoren, alle Rechte vorbehalten)

dargestellt und das in der chirurgischen Notaufnahme praktisch angewandte Vorgehen präsentiert werden (• Abb. 2).

\section{Diagnostik}

Zunächst sollten im Rahmen der initialen Vorstellung, neben der Anamnese, der Tetanusschutz überprüft sowie eine mögliche traumatische Ursache ausgeschlossen werden. Sollte sich in der anschließenden klinischen Untersuchung eine Phlegmone der gesamten Zehe, ein Ulkus oder ein Panaritium zeigen, müssen die Ätiologie identifiziert und entsprechend weitere diagnostische und therapeutische Maßnahmen initiiert werden.

Liegt ein Unguis incarnatus vor, empfehlen wir die Unterscheidung in akute und chronische Formen. In der Literatur wird eine Vielzahl von Klassifikationen postuliert. Die Autoren orientieren sich an den Stadieneinteilungen nach Mozena et al. und Martínez-Nova et al. ([19, 20]; - Tab. 1). Diese basieren auf dem Grad der Inflammation, der Hypertrophie des lateralen Nagelwalls und der Deformität der Nagelplatte.

Als akuten Unguis incarnatus definieren wir das erstmalige Auftreten mit einer Symptomdauer von weniger als 3 Monaten. Dies entspricht den oben genannten Stadien I und II [19, 20]. Entscheidend für die Therapie ist die weitere Subklassifizierung in milde und moderate/schwere Ausprägungsformen (• Abb. 2).

Bei der chronischen Form handelt es sich meist um Rezidive oder den persistierenden Unguis incarnatus mit einer Symptomdauer von mehr als 3 Monaten. Entsprechend den Stadien III und IV $[19,20]$ können hier zusätzlich chroni- sche Deformitäten der Nagelplatte vorliegen. Bei diesen Patienten sehen wir die Durchführung einer Bildgebung als indiziert, um lokale knöcherne Veränderungen, die auf die Nagelmatrix drücken können, zu identifizieren [14].

\section{Therapie}

Die Therapie sollte sich an den oben genannten Stadien orientieren. Die therapeutischen Strategien lassen sich in konservative Maßnahmen, nagelerhaltende Eingriffe und erweiterte chirurgische Maßnahmen untergliedern. Die Therapie hat das Ziel, ein Voranschreiten bzw. eine Chronifizierung zu vermeiden.

\section{Konservative Therapie}

Bei Patienten mit akutem Unguis incarnatus und milder Ausprägung, d.h. ohne Granulationsgewebe, floride Inflammation oder relevanten lokalen Druckschmerz, empfehlen wir die Initiierung der konservativen Therapie (• Abb. 2). Diese beinhaltet die lokale Druckentlastung (Adaptation des Schuhwerks, Zehenzwischenpolsterung) und Nagelpflege, inklusive der Behandlung einer ggf. vorhandenen Onychomykose [18, 21-23]. Durch Fußbäder, bis zu 3-mal tägl., lässt sich nach Einweichen für 10-20 min der Nagelwall von der lateralen Nagelplatte mobilisieren. AnschlieBend können lokal topische Steroide oder desinfizierende Salben über einen Zeitraum von 1 bis 2 Wochen appliziert werden [18]. Zusätzlich kann die laterale Nagelplatte mittels Watte, Gaze oder Kompresse angehoben und abgepolstert werden, um den Druck auf den Nagelwall zu reduzieren (• Abb. 3a; $[18,21])$.
Des Weiteren werden redressierende Tape-Verbände empfohlen, durch die der betroffene Nagelwall mit Pflasterzügeln zur Seite gehalten wird [24]. Allerdings ist die Applikabilität bei aktiven und berufstätigen Patienten limitiert.

Diese Maßnahmen müssen konsequent für 2 bis 12 Wochen, je nach Ausprägung und Therapieansprechen, fortgeführt werden [11, 18]. Dafür empfiehlt sich die Anbindung des Patienten an eine Podologie, die ggf. weitere Maßnahmen, wie z.B. die Anpassung eine Zehenspange, einleiten kann [25].

\section{Operative Therapie}

Bei Patienten mit einem akuten Unguis incarnatus in moderater/schwerer Ausprägung oder mit einem chronischem Unguis incarnatus sollte eine chirurgische Therapie diskutiert werden (- Abb. 2). Die chirurgischen Therapieansätze lassen sich in nagelerhaltende Eingriffe und erweiterte chirurgische Maßnahmen mit dauerhafter Verschmälerung der Nagelplatte untergliedern.

\section{Nagelerhaltende Eingriffe (Notaufnahme)}

Bei Patienten mit akutem Unguis incarnatus in moderater/schwerer Ausprägung, d.h. mit Schmerzen, florider Inflammation und/oder hypertrophem Granulationsgewebe, empfehlen wir die lokale Inspektion und Therapie in Leitungsanästhesie nach Oberst (LA) mit Blutsperre. Dieser Eingriff wird im aseptischen Bereich der Notaufnahme unter sterilen Kautelen durchgeführt. Im klinischen Alltag hat sich die Verwendung eines Handschuhfingers als Blutsperre etabliert [26]. Von einem Klemmen des Gum- 
mizügels wird abgeraten, da durch den hohen Druck Nervenschäden beschrieben sind [27]. Zudem sollte die Größe des Handschuhfingers dem Zehenumfang angepasst werden.

Zuerst sollte das hypertrophe Granulationsgewebe mit einem scharfen Löffel oder dem Skalpell entfernt werden [28]. Anschließend erfolgt die weitere Exploration des Raums zwischen Nagelplatte und Nagelwall. Sollte sich ein abszedierender Verhalt zeigen, kann dieser über denselben Zugang entlastet werden. Parallele Inzisionen am Nagelwall von lateral sollten nicht durchgeführt werden, da die verbleibende, häufig schmale, Hautbrücke nekrotisch werden kann. Zeigen sich in der Exploration Nagelreste oder Fremdkörper, müssen diese entfernt werden. Besonders ist auf ein spitzes laterales Nagelspikulum zu achten. In der Literatur werden in diesem Fall zwei verschiedene Vorgehen beschrieben $[1,11,18]$. Einige Autoren empfehlen die alleinige Glättung des Nagelspikulums, durch Entfernung eines Nagelkeils ( $\bullet$ Abb. 3b). Andere Arbeiten empfehlen die Schienung des lateralen Nagelspikulums mit einem Nagelröllchen (• Abb. 3c; [29]). Dafür kann der Schutzschlauch der Nadel einer ButterflyFlügelkanüle verwendet werden. Dieser sollte proximal abgeschrägt und längs gespalten werden. Anschließend wird die Schiene über das Spikulum und den gesamten Nagelrand geschoben und mittels Naht oder Steri-Strip auf dem Nagel fixiert. So kann die Schiene mit dem Nagel auswachsen [30, 31], der Nagelwall wird geschützt, und die Entzündung klingt ab [21, 31].

Wichtig ist, dass das Spikulum in diesem Fall nicht geglättet wird, da es der Schienung zusätzliche Stabilität verleiht [21, 31].

Für die alleinige Entfernung des Granulationsgewebes und die anschließende Glättung des lateralen Nagelspikulums sind Rezidivraten bis zu $30-70 \%$ beschrieben $[1,32]$. Da hier die sorgfältige Entfernung des lateralen Matrixhorn und der Nagelmatrix ausbleibt, kann die Nagelplatte weiter entlang des Nagelwalls wachsen und zur ständigen Irritation führen [33]. Für die Schienung mittels Nagelröllchen sind deutlich geringere Rezidivraten bis $\mathrm{zu} 10 \%$ beschrieben

Unfallchirurg 2021 · 124:311-318 https://doi.org/10.1007/s00113-020-00903-6

(c) Der/die Autor(en) 2020

N. Moellhoff · H. Polzer · S. F. Baumbach · K. G. Kanz · W. Böcker · V. Bogner-Flatz

Unguis incarnatus - konservative oder operative Therapie? Ein praktischer Behandlungsalgorithmus

\section{Zusammenfassung}

Der Unguis incarnatus ist ein häufiges Krankheitsbild, mit dem sich Patienten in der Hausarztpraxis, der dermatologischen Klinik oder der chirurgischen Notaufnahme vorstellen. Häufig führt die inkonsequente konservative Therapie oder die falschindizierte operative Intervention zu langwierigen und komplikationsreichen Verläufen, inklusive Rezidiven. Die Patienten sollten über die Komplexität des Nagelorgans aufgeklärt werden, um der Banalisierung der Erkrankung vorzubeugen, und eine entsprechende Compliance in der Therapie zu erreichen. In diesem Manuskript wird die sachgerechte Versorgung des Unguis incarnatus im Sinne eines praktischen Behandlungsalgorithmus dargestellt. Die konsequente konservative
Therapie ist bei akutem Unguis incarnatus mit milder Ausprägung die Therapie der ersten Wahl mit guten Behandlungsergebnissen. Nagelerhaltende operative Eingriffe kommen bei moderaten/schweren akuten Formen zum Einsatz. Der chronische Unguis incarnatus, ohne floride Infektion, stellt eine elektive Operationsindikation dar. Sowohl bei den nagelerhaltenden Eingriffen als auch bei erweiterten operativen Maßnahmen ist eine chirurgische Operationsaufklärung obligat.

\section{Schlüsselwörter}

Eingewachsener Zehennagel · Onychocryptosis - Emmert-Plastik · Podologie . Phenolkauterisierung

\section{Unguis incarnatus_-conservative or operative treatment? A practical treatment algorithm}

\section{Abstract}

Unguis incarnatus, an ingrown toenail, is a common condition in primary care, which is encountered by various medical professions. Inconsistent conservative treatment and nonindicated surgical treatment often result in complications and recurrence of the disease. Patients must be thoroughly informed about the complexity of the nail organ. This is a prerequisite to prevent trivialization of the disease and to achieve appropriate patient compliance for treatment. In this article a practical diagnostic and treatment algorithm for unguis incarnatus is presented. In mild cases of acute unguis incarnatus a consistent conservative treatment is the first-line strategy showing promising results. In cases of moderate to severe forms of acute unguis incarnatus, surgical procedures that preserve the nail matrix should be applied. For cases of chronic unguis incarnatus without an acute infection, elective partial matrixectomy can be indicated. Prior to any surgical intervention, detailed informed consent must be obtained from the patients.

\section{Keywords}

Ingrown toenail · Onychocryptosis · Emmertplasty · Podiatry · Phenol cauterization
[30, 34, 35]. Jedoch ist der Erfolg stark von der Compliance der Patienten abhängig.

\section{Erweiterte operative Maßnahmen (Operationssaal)}

Bei Patienten mit chronischem Unguis incarnatus ist nur bei florider Infektion eine sofortige Exploration indiziert (๑ Abb. 2). Liegt diese nicht vor, sollte das weitere Vorgehen in enger Kooperation mit der Fußchirurgie abgestimmt werden. Hier bedarf es häufig erweiterter operativer Maßnahmen, die durch dau- erhafte Verschmälerung der Nagelplatte das Einwachsen des Nagels verhindern. Diese Eingriffe sollten durch einen Facharzt im Rahmen einer elektiven ambulanten Operation durchgeführt werden.

In Deutschland kommt immer noch führend die Nagelkeilresektion (z.B. nach Emmert) zur Anwendung (- Abb. 3d; [36]). Auch diese wird, ebenso wie die oben beschriebenen nagelerhaltenden Eingriffe, in LA und lokaler Blutsperre durchgeführt. Bei der Nagelkeilresektion erfolgen das Ausschneiden des erkrankten randständigen Zehen- 


\section{Originalien}

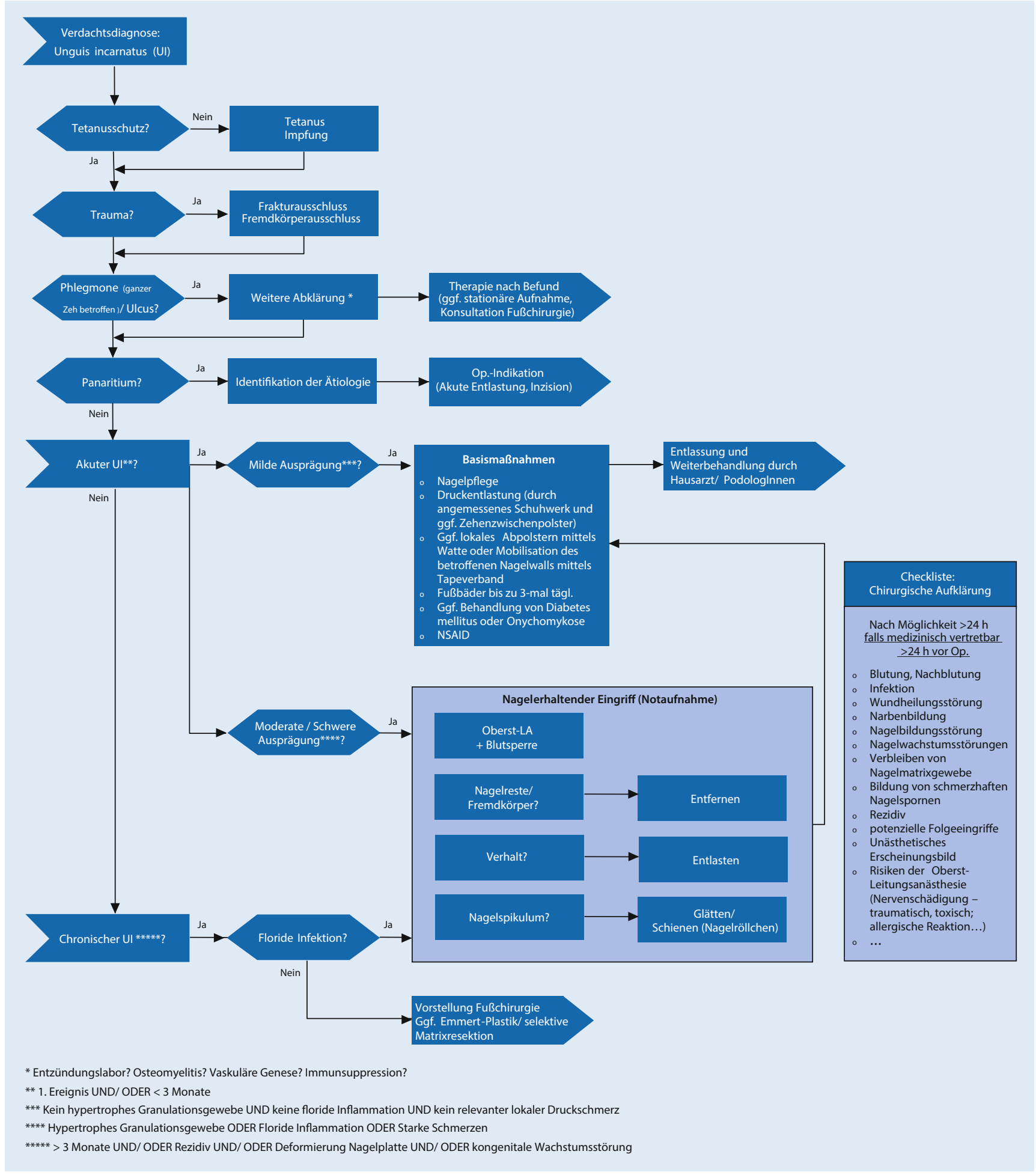

Abb. $2 \Delta$ Behandlungsalgorithmus zu Diagnostik und Therapie des Unguis incarnatus. UI Unguis incarnatus, LA Leitungsanästhesie, $h$ hours (Stunden), NSAID non-steroidal anti-inflammatory drug. (Mit freundl. Genehmigung der Autoren, alle Rechte vorbehalten) 
Tab. 1 Stadieneinteilung des Unguis incarnatus. (Modifiziert nach Mozena et al. [19] und Martínez-Nova et al. [20])

\begin{tabular}{ll}
$\begin{array}{l}\text { Stadien- } \\
\text { einteilung }\end{array}$ & Klinisches Bild \\
\hline I & $\begin{array}{l}\text { Erythem, Ödem, Schmerz bei Druck auf lateralen Nagelwall, keine Wucherung des } \\
\text { Nagelwalls über die Nagelplatte }\end{array}$ \\
Ila & $\begin{array}{l}\text { Symptome wie in I, vermehrte Schmerzen, Ödem, Erythem, Hyperästhesie, seröses } \\
\text { Wundexsudat, ggf. Infektion, Wucherung des Nagelwalls über die Nagelplatte } \\
<3 \mathrm{~mm}\end{array}$ \\
IIb & $\begin{array}{l}\text { Symptome wie in Ila, ggf. Infektion und Abszedierung mit Wucherung des Nagel- } \\
\text { walls über die Nagelplatte }>3 \text { mm }\end{array}$ \\
III & $\begin{array}{l}\text { Chronische Hypertrophie und Hypergranulation, Gewebe bedeckt einen Großteil } \\
\text { der Nagelplatte, ggf. Abszess, Ulzeration, Deformierung }\end{array}$ \\
IV & $\begin{array}{l}\text { Chronische Deformierung der Nagelplatte, beider lateraler Nagelwälle, Hypertro- } \\
\text { phie mit Bildung eines distalen Nagelwalls }\end{array}$
\end{tabular}

nagelviertels sowie die Keilexzision von Nagelwall und Nagelbett mit vollständiger Entfernung der Nagelmatrix bis auf die Endphalanx der Zehe [36]. Allerdings sind Rezidivraten bis zu $20 \%$ sowie schlechte ästhetische und funktionelle Ergebnisse beschrieben [37-39]. Ursächlich für die hohe Rezidivrate sind a.e. die unvollständige Resektion des lateralen Matrixhorns und der lateralen Nagelmatrix durch die keilförmige Exzision. Dies führt zu bizarren und schmerzhaften Nagelspikula. Des Weiteren kann es zu Nagelplattendeviation in Narbenrichtung kommen. Weiterhin problematisch sind die oft ausgeprägten postoperativen Schmerzen sowie mögliche Wundheilungsstörungen. Hier sind Infektionsraten bis zu $20 \%$ beschrieben $[5,21,40]$. Aus diesen Gründen wird diese Operationstechnik kritisch diskutiert [21, 37-39, 41-43].

Heutzutage wird von vielen Autoren die schonendere selektive Nagelmatrixablation als Behandlungsstandard angesehen [11, 17, 18, 21, 39, 40, 44-46]. Das Ziel ist die selektive Entfernung des lateralen Nagelrands, inklusive des Matrixhorns und der dazugehörigen Nagelmatrix. Im Gegensatz zur Emmert-Plastik erfolgt keine Entfernung des Nagelwalls. Für die selektive Nagelmatrixablation sind zwei verschiedene Techniken beschrieben: die chirurgische oder chemische Ablation der Nagelmatrix.

Bei der chirurgischen Ablation erfolgt, analog zur Emmert-Plastik, das Setzen eines Hautschnitts in Verlängerung des Nagelwalls nach proximal bis kurz vor das (distale) Interphalangealgelenk
(- Abb. 3e). Alternativ kann die Inzision nach lateral geschwungen durchgeführt werden [21]. Anschließend wird der Nagel vom lateralen Nagelwall und vom Nagelbett gelöst. Dann erfolgt die Identifikation der Grenze zwischen gesundem und erkranktem Nagel. An dieser Grenze wird an der distalen Nagelplatte das Skalpell (15er-Klinge) mit nach oben gerichteter Klinge angesetzt. Dann wird das Skalpell, geführt durch die Nagelfaserrichtung, über die zuvor durchgeführte Hautinzision, bis an das proximale Ende der Nagelmatrix gedrückt. Dadurch wird zum einen das Nagelbett geschont, zum anderen wird der $\mathrm{zu}$ resezierende laterale Nagelteil, inklusive Nagelhorn, klar definiert. Dieser Nagelstreifen wird in toto entfernt. Schließlich erfolgt das sorgsame Entfernen des dazugehörigen Nagelmatrixepithels mit dem scharfen Löffel. Der proximale Hautschnitt wird mit 2 Einzelknopfnähten verschlossen.

Bei der chemischen Ablation der Nagelmatrix kann auf den proximalen Hautschnitt vollständig verzichtet werden (-Abb. 3f). Nach Mobilisation des lateralen Nagelwalls wird nun das proximale Nagelhorn luxiert. Anschließend wird die Grenze zwischen gesundem und erkranktem Nagel definiert und, analog zu der chirurgischen Exzision, der erkrankte Nagelstreifen reseziert. Das Nagelmatrixepithel wird nun nicht chirurgisch, sondern chemisch abladiert. Dafür wird zuerst der proximale Nagelwall stumpf mobilisiert und anschließend das Nagelmatrixepithel mit in Phenol getränkten sterilen Wattestäbchen abladiert. Das Nagelmatrixepithel wird unter drehenden Bewegungen für je 3-mal 1 min unter Druck mit dem Wattestäbchen eingerieben [5, 47]. Dabei sollten die Drehbewegungen von der Nagelplatte weg, hin zum ipsilateralen Nagelwall erfolgen, um eine Schädigung der medialen Nagelmatrix sowie des Restnagels zu verhindern. Die Phenolisierung verursacht eine kontrollierte Nekrose des Matrixepithels und des darunter liegenden Bindegewebes [21]. Der Situs muss zum Zeitpunkt der Phenolapplikation bluttrocken sein, da das Phenol sonst lediglich die Blutproteine koaguliert und seine Wirkung nicht am Matrixepithel entfaltet. Abschließend folgt die Spülung des Situs mit NaCl-, Ringer-Lösung oder medizinischem Alkohol zur Verdünnung des Phenols am Auftragungsort [48].

In der Literatur wird die Überlegenheit einer der beiden Methoden kontrovers diskutiert. Mehrere Studien konnten zeigen, dass die chirurgische Resektion zwar zu weniger Rezidiven führt, die chemische Ablation aber insgesamt bessere postoperative Ergebnisse erzielt [44, 49]. Die Rezidivraten werden meistens zwischen 5 und $18 \%$ angegeben $[18,44,45$, 49-52]. Ein Cochrane Review aus 2012 von Eekhof et al. (24 Studien, 2826 Patienten) kam, allerdings bei eingeschränkter Studienlage, zu dem Schluss, dass die chemische Ablation der chirurgischen Resektion hinsichtlich der Rezidivrate überlegen ist [17]. Allerdings kritisieren einige Autoren, dass die chemische Ablation in stark sezernierenden Wunden resultieren kann. Dies ist wiederrum mit einer erhöhten Infektionsrate assoziiert $[40,53]$. Zudem wird kontrovers diskutiert, ob die Phenolapplikation ein gesundheitliches Risiko für den Anwender darstellt [54-56]. Aus diesen Gründen empfehlen wir, auch aufgrund der Tatsache der ausgewiesenen chirurgischen Expertise an unserem Haus, die selektive chirurgische Nagelmatrixresektion.

Zahlreiche weitere Verfahren sind beschrieben, werden in der Routine jedoch nicht eingesetzt. Beispielsweise erfolgt der Einsatz von $\mathrm{CO}_{2}$-Lasern und Elektrokautern zur Nagelmatrixentfernung [11, 18, 57-59]. Auch kann der laterale Nagelwall elliptisch oder halbmondförmig exzidiert und readaptiert werden. 


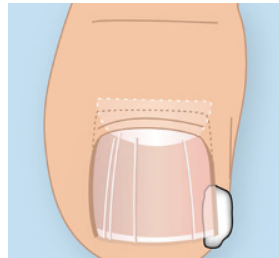

a

Abb. $3 \Delta$ Konservative und operative Behandlungsverfahren. a Anhebung der Nagelplatte mittels gerollter Gaze, b partielle Nagelentfernung und Glättung des Nagelspikulums, c Schienung der Nagelplatte und des Nagelspikulums mittels längs aufgeschnittenem Butterfly-Schlauch, d Keilresektion, e selektive chirurgische Nagelmatrixablation, fselektive Nagelmatrixablation mit Phenol. (a-d,f aus Khalil [33], Gestaltung und Layout sowie Modifikation von H. Thun, Stabsstelle Kommunikation und Medien, Klinikum der Universität München; mit freundl. Genehmigung; e mit freundl. Genehmigung der Autoren, alle Rechte vorbehalten)

Dadurch wird eine plane Fläche und damit Raum für die laterale Nagelplatte geschaffen [60, 61]. Totale Nagelextraktionen sind beschrieben, werden heutzutage aber nicht mehr empfohlen [21].

\section{Additive Antibiotika}

Die Notwendigkeit einer additiven Antibiose wird regelmäßig diskutiert. Allerdings konnten mehrere Studien zeigen, dass weder die orale noch die topische Antibiotikatherapie einen Einfluss auf das Behandlungsergebnis haben [4, $11,17]$. Wir verwenden keinerlei topische Antibiose. Die orale Antibiose sehen wir lediglich bei ausgewählten Fällen mit putridem, abszedierenden Geschehen als indiziert an. In diesen Fällen sollten Antibiotika mit einer guten Gewebegängigkeit, z.B. Clindamycin, verwendet werden [62].

\section{Aufklärung}

Die Dokumentation und Aufklärung haben einen zentralen Stellenwert in der Therapie. Bei der konservativen Therapie sollten die Maßnahmen und Dauer ausführlich dokumentiert werden. Sowohl bei den nagelerhaltenden Eingriffen als auch bei den erweiterten operativen Maßnahmen ist eine Aufklärung zwingend notwendig. Sofern es der medizinische Befund zulässt, müssen die geforderten medikolegalen Zeitfristen der chirurgischen Aufklärung, die mindestens 24Stunden vor dem Eingriff erfolgen soll, eingehalten werden. Die Aufklärung sollte, unabhängig vom
Operationsverfahren, folgende Punkte beinhalten: Blutung und Nachblutung, Infektion, Wundheilungsstörung, Nagelbildungs- und Nagelwachstumsstörung, dauerhafte Verschmälerung der Nagelplatte, das Verbleiben von Nagelmatrixgewebe und die Bildung von schmerzhaften Nagelspornen, Rezidive, Folgeeingriffe, Narbenbildung und ein resultierendes unästhetisches Erscheinungsbild. Über mögliche Nervenverletzungen (traumatisch, toxisch) sowie allergische Reaktionen durch die Leitungsanästhesie sollte ebenfalls aufgeklärt werden.

\section{Schlussfolgerung}

Der Unguis incarnatus ist ein häufiges Krankheitsbild. Die Therapie umfasst konservative sowie operative Maßnahmen. Diese sollten stadiengerecht angewandt werden. Essenziell für die erfolgreiche Behandlung des Unguis incarnatus ist die korrekte Indikationsstellung. Im Rahmen dieser Arbeit wird ein entsprechender praktischer Diagnoseund Behandlungsalgorithmus postuliert (- Abb. 2). Die konservative Therapie sollte bei akutem Unguis incarnatus mit milder Ausprägung konsequent durchgeführt werden und zeigt gute Behandlungsergebnisse. Nagelerhaltende Eingriffe kommen bei moderaten/schweren Formen zum Einsatz. Der chronische Unguis incarnatus ohne floride Infektion stellt eine elektive Operationsindikation dar. In den meisten Fällen führen wir die selektive chirurgische Nagelmatrixresektion durch. Bei allen chirurgischen
Maßnahmen muss die konsequente chirurgische Aufklärung erfolgen.

\section{Fazit für die Praxis}

\section{- Der eingewachsene Zehennagel ist keine Banalität. \\ - Bei akutem Unguis incarnatus mit milder Ausprägung sollte die konser- vative Therapie erfolgen. \\ - Bei akutem Unguis incarnatus mit moderater/schwerer Ausprägung werden nagelerhaltende Eingriffe in Lokalanästhesie empfohlen. \\ - Chronische Fälle ohne floride Infekti- on erfordern erweiterte chirurgische Maßnahmen. \\ - Die Emmert-Plastik wurde in den meisten Fällen durch die chirurgische oder chemische Nagelmatrixablation ersetzt. \\ - Jeder chirurgische Eingriff bedarf einer suffizienten chirurgischen Aufklärung.}

\section{Korrespondenzadresse}

\section{N. Moellhoff}

Abteilung für Hand-, Plastische und Ästhetische Chirurgie, Klinikum der Universität München, LMU München

Pettenkoferstr. 8a, 80336 München,

Deutschland

nicholas.moellhoff@med.uni-muenchen.de

Funding. Open Access funding enabled and organized by Projekt DEAL. 


\section{Einhaltung ethischer Richtlinien}

Interessenkonflikt. N. Moellhoff, H. Polzer, S.F. Baumbach, K.G. Kanz, W. Böcker und V. Bogner-Flatz geben an, dass kein Interessenkonflikt besteht.

\section{Für diesen Beitrag wurden von den Autoren keine} Studien an Menschen oder Tieren durchgeführt Für die aufgeführten Studien gelten die jeweils dort angegebenen ethischen Richtlinien.

Open Access. Dieser Artikel wird unter der Creative Commons Namensnennung 4.0 International Lizenz veröffentlicht, welche die Nutzung, Vervielfältigung, Bearbeitung, Verbreitung und Wiedergabe in jeglichem Medium und Format erlaubt, sofern Sie den/die ursprünglichen Autor(en) und die Quelle ordnungsgemäß nennen, einen Link zur Creative Commons Lizenz beifügen und angeben, ob Änderungen vorgenommen wurden.

Die in diesem Artikel enthaltenen Bilder und sonstiges Drittmaterial unterliegen ebenfalls der genannten Creative Commons Lizenz, sofern sich aus der Abbildungslegende nichts anderes ergibt. Sofern das betreffende Material nicht unter der genannten Creative Commons Lizenz steht und die betreffende Handlung nicht nach gesetzlichen Vorschriften erlaubt ist, ist für die oben aufgeführten Weiterverwendungen des Materials die Einwilligung des jeweiligen Rechteinhabers einzuholen.

Weitere Details zur Lizenz entnehmen Sie bitte der Lizenzinformation auf http://creativecommons.org/ licenses/by/4.0/deed.de.

\section{Literatur}

1. Zuber TJ (2002) Ingrown toenail removal. Am Fam Physician 65:2547-2552 (2554)

2. Haneke E (2014) Anatomie, Biologie, Physiologie und Grundzüge der Pathologie des Nagelorgans. Hautarzt 65:282-290

3. Wollina U, Nenoff P, Haroske G, Haenssle HA (2016) The diagnosis and treatment of nail disorders. Dtsch Arztebl Int 113:509-518

4. Reyzelman AM, Trombello KA, Vayser DJ, Armstrong DG, Harkless LB (2000) Are antibiotics necessary in the treatment of locally infected ingrown toenails? Arch Fam Med 9:930-932

5. Mainusch OM, Loser CR (2018) Ingrown toenailsoptions for daily practice. Hautarzt 69:726-730

6. Levy LA (1992) Prevalence of chronic podiatric conditions in the US. National Health Survey 1990. J Am Podiatr Med Assoc 82:221-223

7. Cho SY, Kim YC, Choi JW (2018) Epidemiology and bone-related comorbidities of ingrown nail: a nationwide population-based study. J Dermatol 45:1418-1424

8. DeLauro NM, DeLauroTM (2004) Onychocryptosis. Clin Podiatr Med Surg 21:617-630 (vii)

9. Ezekian B et al (2017) Onychocryptosis in the pediatric patient. Clin Pediatr (Phila) 56:109-114

10. Heidary N, Naik H, Burgin S (2008) Chemotherapeutic agents and the skin: an update. J Am Acad Dermatol 58:545-570

11. Heidelbaugh JJ, Lee $H$ (2009) Management of the ingrown toenail. Am Fam Physician 79:303-308

12. Rauch C, Cherkaoui-Rbati M (2014) Physics of nail conditions: why do ingrown nails always happen in the big toes? Phys Biol 11:66004
13. Kose O, Celiktas M, Kisin B, Ozyurek S, Yigit S (2011) Is there a relationship between forefoot alignment and ingrown toenail? A case-control study. Foot Ankle Spec 4:14-17

14. Baran R, Haneke E, Richert B (2001) Pincer nails: definition and surgical treatment. Dermatol Surg 27:261-266

15. Freiberg A, Dougherty S (1988) A review of management of ingrown toenails and onychogry phosis. Can Fam Physician 34:2675-2681

16. Langford DT, Burke C, Robertson K (1989) Risk factors in onychocryptosis. Br J Surg 76:45-48

17. Eekhof JAH, Van Wijk B, Knuistingh Neven A, van der Wouden JC (2012) Interventions for ingrowing toenails. Cochrane Database Syst Rev 4:CD1541

18. Mayeaux EJ Jr, Carter C, Murphy TE (2019) Ingrown toenail management. Am Fam Physician 100:158-164

19. Mozena JD (2002) The Mozena classification system and treatment algorithm for ingrown hallux nails. J Am Podiatr Med Assoc 92:131-135

20. Martinez-Nova A, Sanchez-Rodriguez R, AlonsoPena D (2007) A new onychocryptosis classification and treatment plan. J Am Podiatr Med Assoc 97:389-393

21. Haneke E (2012) Controversies in the treatment of ingrown nails. Dermatol Res Pract 2012:783924

22. Connolly B, Fitzgerald RJ (1988) Pledgets in ingrowing toenails. Arch Dis Child 63:71-72

23. Senapati A (1986) Conservative outpatient management of ingrowing toenails. J R Soc Med 79:339-340

24. Nishioka K, Katayama I, Kobayashi Y, Takijiri C, Nishioka K (1985) Taping for embedded toenails. $\mathrm{Br}$ J Dermatol 113:246-247

25. Chiriac A, Solovan C, Brzezinski P (2014) Ingrown toenails (unguis incarnatus): nail braces/bracing treatment. Proc (Bayl Univ Med Cent) 27:145

26. Osanai T, Ogino T (2010) Modified digital tourniquet designed to prevent the tourniquet from inadvertently being left in place after the end of surgery. J Orthop Trauma 24:387-388

27. Lahham $S$ et al (2011) Comparison of pressures applied by digital tourniquets in the emergency department. West JEmerg Med 12:242-249

28. Kang MH et al (2008) The effect of removal of granulation tissue on ingrown toenails associated with granulation tissue. Korean J Dermato 46:453-458

29. Wallace WA, Milne DD, Andrew T (1979) Gutter treatment for ingrowing toenails. Br Med J 2:168-171

30. Nazari S (2006) A simple and practical method in treatment of ingrown nails: splinting by flexible tube. J Eur Acad Dermatol Venereol 20:1302-1306

31. Arai $H$, Arai T, Nakajima H, Haneke E (2004) Formable acrylic treatment for ingrowing nail with gutter splint and sculptured nail. Int J Dermatol 43:759-765

32. Palmer BV, Jones A (1979) Ingrowing toenails: the results of treatment. Br JSurg 66:575-576

33. Khalil PN (2010) Was geht konservativ, was nur mit Skalpell? MMW Fortschr Med 152:46-48

34. Kim YJ, Ko JH, Choi KC, Lee CG, Lim KJ (2003) Nail-splinting technique for ingrown nails: the therapeutic effects and the proper removal time of the splint. Dermatol Surg 29:745-748

35. Schulte KW, Neumann NJ, Ruzicka T (1998) Surgical pearl: nail splinting by flexible tube-a new noninvasive treatment for ingrown toenails. J Am Acad Dermatol 39:629-630

36. Strube H-D, Wasserscheid B (1990) Emmert-Plastik beim Unguis incarnatus. Operat Orthop Traumatol 2:39-45
37. Kose 0 et al (2012) Cosmetic results of wedge resection of nail matrix (Winograd technique) in the treatment of ingrown toenail. Foot Ankle Spec $5: 241-244$

38. Noel B (2008) Surgical treatment of ingrowntoenail without matricectomy. Dermatol Surg 34:79-83

39. Rounding C, Bloomfield S (2005) Surgical treatments for ingrowing toenails. Cochrane Database Syst Rev 2:CD1541

40. Richert $B$ (2012) Surgical management of ingrown toenails - an update overdue. Dermatol Ther 25:498-509

41. Haneke E (2015) Nail surgery. In: Robinson JK, Hanke CW, Siegel DM, Fratila A (Hrsg) Surgery of the skin, Bd. 3. Elsevier, Amsterdam, S767

42. Haneke E, Baran RL (1993) Nail surgery. In: Harahap M (Hrsg) Complications of dermatologic surgery, Bd. 1.Springer, Berlin, S88-89

43. Haneke E (2016) Nail surgery. In: Andre P, Haneke E, Marini L, Payne CR (Hrsg) Cosmetic medicine and surgery, Bd. 1. Taylor \& Francis, Boca Raton

44. HasselJC, Hassel AJ, Loser C(2010) Phenol chemical matricectomy is less painful, with shorter recovery times but higher recurrence rates, than surgical matricectomy: a patient's view. Dermatol Surg 36:1294-1299

45. Andreassi A, Grimaldi L, D’Aniello C, Pianigiani E, Bilenchi R (2004) Segmental phenolization for the treatment of ingrowing toenails: a review of 6 years experience. J Dermatolog Treat 15:179-181

46. Haneke E (2013) Nail surgery. Clin Dermatol 31:516-525

47. Tatlican S, Yamangokturk B, Eren C, Eskioglu F, Adiyaman S (2009) Comparison of phenol applications of different durations for the cauterization of the germinal matrix: an efficacy and safety study. Acta Orthop Traumatol Turc 43:298-302

48. Burzotta JL, Turri RM, Tsouris J (1989) Phenol and alcohol chemical matrixectomy. Clin Podiatr Med Surg 6:453-467

49. Romero-Pérez D, Betlloch-Mas I, Encabo-Durán B (2017) Onychocryptosis: a long-term retrospective and comparative follow-up study of surgical and phenol chemical matricectomy in 520 procedures. Int JDermatol 56:221-224

50. Di Chiacchio N, Di Chiacchio NG (2015) Best way to treat an ingrown toenail. Dermatol Clin 33:277-282

51. Bostanci S, Ekmekçi P, Gürgey E (2001) Chemical matricectomy with phenol for the treatment of ingrowing toenail: a review of the literature and follow-up of 172 treated patients. Acta Derm Venereol 81:181-183

52. Di Chiacchio N, Belda W Jr, Di Chiacchio NG, Kezam Gabriel FV, de Farias DC (2010) Nail matrix phenolization for treatment of ingrowing nail: technique report and recurrence rate of 267 surgeries. Dermatol Surg 36:534-537

53. Gilles GA, Dennis KJ, Harkless LB (1986) Periostitis associated with phenol matricectomies. J Am Podiatr Med Assoc 76:469-472

54. Losa Iglesias ME et al (2008) Safety of phenol vapor inhalation during performance of chemical matrixectomy to treat ingrown toenails. Dermatol Surg 34:1515-1519

55. Sugden P, Levy M, Rao GS (2001) Onychocryptosisphenol burn fiasco. Burns 27:289-292

56. Warner MA, Harper JV (1985) Cardiac dysrhythmias associated with chemical peeling with phenol. Anesthesiology 62:366-367

57. Apfelberg DB, Rothermel E, Widtfeldt A, Maser MR, Lash H (1984) Preliminary report on use of carbon dioxide laser in podiatry. J Am Podiatry Assoc 74:509-513 
58. Tada H et al (2004) Clinical comparison of the scanning $\mathrm{CO} 2$ laser and conventional surgery in the treatment of ingrown nail deformities. JDermatolog Treat 15:387-390

59. Yang KC, Li YT (2002) Treatment of recurrent ingrown great toenail associated with granulation tissue by partial nail avulsion followed by matricectomy with sharpulse carbon dioxide laser. Dermatol Surg 28:419-421

60. Bose B (1971) A technique for excision of nail fold for ingrowing toenail. Surg Gynecol Obstet 132:511-512

61. Sarifakioglu E, Sarifakioglu N (2010) Crescent excision of the nail fold with partial nail avulsion does work with ingrown toenails. Eur J Dermatol 20:822-823

62. Langer MF, Wieskötter B, Oeckenpöhler S, Breiter S (2014) Akute Infektionen im Bereich des Fingernagels - die akuten Paronychien. Handchir Scan 3:69-85

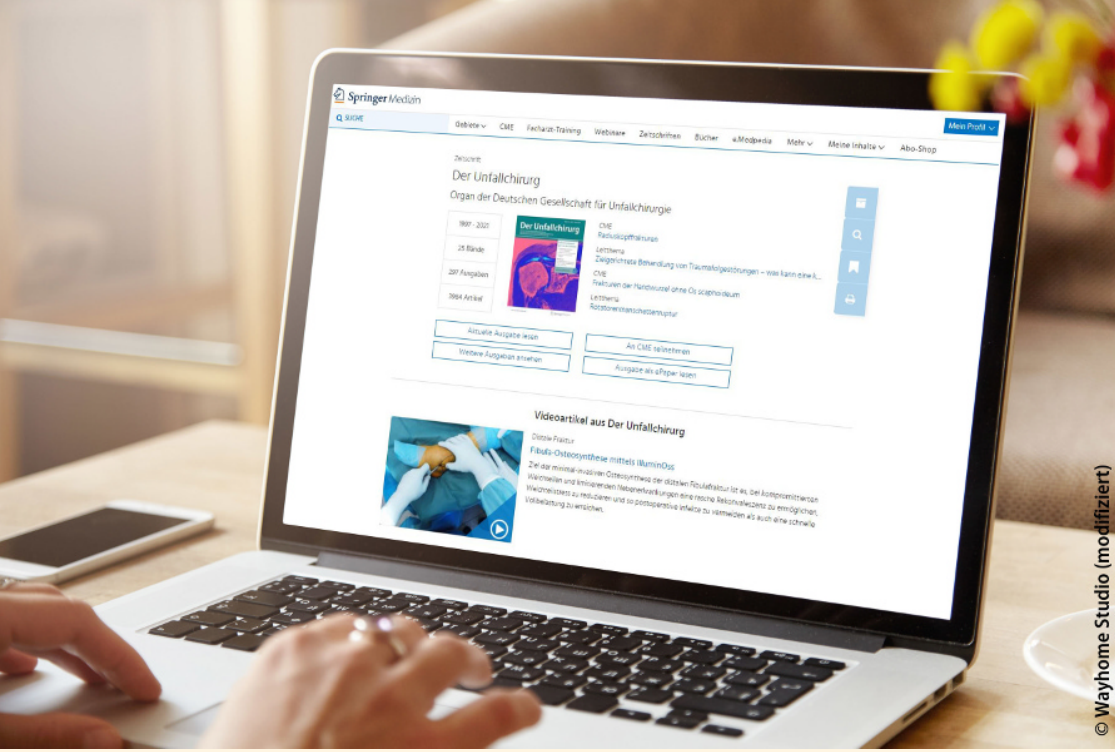

Auch online Zugang zu allen Beiträgen von Der Unfallchirurg

Wussten Sie, dass Sie als Abonnent*in dieser Zeitschrift automatisch online Zugriffsrechte auf das gesamte Beitragsarchiv haben?

Durch Ihr Abonnement von Der Unfallchirurg erhalten Sie jeden Monat die aktuelle Ausgabe der Zeitschrift nach Hause geliefert. Doch damit nicht genug: Sie haben mit dem Abonnement außerdem Zugriff auf das gesamte Online-Archiv Ihrer Zeitschrift.

Und so einfach geht es:

Registrieren Sie sich einmal über www.springermedizin.de/register:

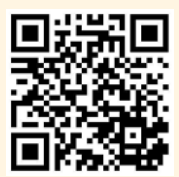

Über diesen QR-Code schnell und einfach registrieren

Bei der Registrierung geben Sie einfach Ihren Vor- und Nachname und Lieferadresse wie beim Abonnement der Zeitschrift (siehe Adressaufkleber auf Ihrem Heft) an. So kann im System die Zugehörigkeit zu Ihrer Zeitschrift sichergestellt werden.

Sollten Fragen oder Probleme auftauchen, wenden Sie sich einfach an den Kundenservice:

kundenservice@springermedizin.de
Aufgrund des Heilmittelwerbegesetzes dürfen die Inhalte der Website nur medizinischen Fachkreisen zur Verfügung gestellt werden. Bei der Anmeldung bitten wir Sie deshalb einen Berufsnachweis vorzulegen. Bei Mediziner*innen mit Mitgliedschaft in der deutschen Ärztekammer reicht die einheitliche Fortbildungsnummer (EFN). Als Angehörige ${ }^{*} r$ eines medizinischen Berufs schicken Sie eine Bestätigung der Arbeitsstelle. Sind Sie Student*in, dann senden Sie bitte den Studiennachweis mit Angabe des Studiengangs ganz unkompliziert an: kundenservice@springermedizin.de.

Mit Benutzername und Passwort haben Sie außerdem Zugang zu den freien Inhalten auf den Seiten von: https://www.springermedizin.de/ https://www.aerztezeitung.de/ 